\title{
Identification of Potential Biomarkers for Giant Cell Tumor of Bone Using Comparative Proteomics Analysis
}

\author{
Amalia Conti, ${ }^{*}$ Gemma Caballero Rodriguez, ${ }^{\dagger}$ \\ Antonella Chiechi, ${ }^{*}$ Rosa Maria Dégano Blazquez, ${ }^{\dagger}$ \\ Victoria Barbado, ${ }^{\dagger}$ Tibor Krènacs, ${ }^{\ddagger}$ Chiara Novello, ${ }^{*}$ \\ Laura Pazzaglia, ${ }^{*}$ Irene Quattrini, ${ }^{,}$Licciana Zanella, ${ }^{\S}$ \\ Piero Picci, ${ }^{*}$ Enrique De Alava ${ }^{\dagger}{ }^{,}$and \\ Maria Serena Benassi*
}

one peroxidase 1 was strongly related to local recurrence and metastasis, suggesting that its up-regulation may identify a subset of high-risk patients with giant cell tumor prone to receive diverse clinical management. (Am J Patbol 2011, 178:88-97; DOI: 10.1016/j.ajpath.2010.11.035)

Giant cell tumor (GCT) is a benign bone tumor with fairly high local aggressiveness, and development of lung metastases is rare, occurring in $2 \%$ to $5 \%$ of cases. ${ }^{1}$ Histologically, the tumor pattern is formed by a network of spindle-shaped mononuclear stroma cells, round mononuclear histiocytic cells, and multinuclear giant cells similar to osteoclasts. ${ }^{2}$

Cellular components interact with various factors playing a role in osteoclast function regulation. In fact, precursors of osteoclasts express receptor activator of $\mathrm{NF}-\kappa \mathrm{B}$ that in the presence of macrophage colonystimulating factor and its ligand, receptor activator of $\mathrm{NF}-\kappa \mathrm{B}$ ligand, mediates osteoclast formation by increasing the expression of enzymes that dissolve organic and inorganic components of bone., ${ }^{3,4}$ At the same time, the endogenous osteoprotegerin counteracts these effects by competing for receptor activator of NF- $\kappa \mathrm{B}$ ligand and neutralizing it. These interactions may provide information to help develop new approaches to biological therapy of this tumor. Drugs that target the osteolytic process lower recurrence rates associated with morbidity and mortality and are considered useful for new clinical treatments. 5,6

\footnotetext{
Supported by grants from the Italian Ministry of Health (Programma straordinario Ricerca Oncologica) and Istituto Superiore della Sanità (Italia-USA Project and ACC10). Istituto Ortopedico Rizzoli, Centro de Investigación del Cancer-IBMCC and Semmelweis University are partners of the EuroBoNeT consortium, a network of excellence granted by the European Commission for studying the pathology and genetics of bone tumors.

Accepted for publication September 28, 2010.

Address reprint requests to Maria Serena Benassi, Ph.D., Laboratory of Experimental Oncology, Istituto Ortopedico Rizzoli, Via di Barbiano 1/10, 40136 Bologna, Italy. E-mail: mariaserena.benassi@ior.it.
} 
There are many hypotheses regarding relapsed GCTs and their biological behavior. Cytogenetically, the most common chromosome aberrations are telomeric associations involving multiple chromosome ends that are responsible for structural chromosomal aberrations, an important event in GCT recurrences. ${ }^{7}$ Moreover, a previous study demonstrated that amplification of the 29q11.1 chromosome region in a series of GCTs was associated with local or systemic relapse. ${ }^{8}$

The rarity of GCT results in a paucity of human tumor specimens for analyses, but recently, the development of high throughput screening techniques has allowed global investigations of the molecular background of individual patient tumor samples, providing data for the selection of biomarkers with significant clinical impact. ${ }^{9}$

Microarray analysis identified a list of tumor-related genes associated with GCT pathogenesis and development, ${ }^{10-12}$ but few studies have correlated gene/protein differential expression with clinical data. ${ }^{13} \mathrm{~A}$ recent study provided evidence of the clinical impact of global protein expression studies to identify new diagnostic and prognostic factors in osteosarcoma and soft tissue sarcomas, revealing a cluster of proteins according to histologic type and chemosensitivity. ${ }^{14}$

Because proteomics studies can recognize and identify specifically expressed molecules and deregulated pathways associated with different states of tumor progression, we conducted a study using proteomics approaches with the aim of identifying a list of candidate prognostic biomarkers useful for stratifying patients with GCT according to their risk of local or distant relapse.

\section{Materials and Methods}

The research protocol was approved by the ethics committee of the Orthopedic Rizzoli Institute where the study was started, and all the patients provided appropriate informed consent. Only entities with classic GCT of bone were included in the study. All histologic slides were reviewed, and diagnosis was confirmed by pathologists with expertise in bone tumors (T.K. and P.P.). The 155 selected patients had complete clinical medical records. ${ }^{15}$ Of the total cohort of patients, 83 were
Table 1. Clinical Characteristics of 155 Patients with Primary GCT

\begin{tabular}{lc}
\hline \multicolumn{1}{c}{ Characteristics } & Values \\
\hline Sex, no. & \\
Male & 80 \\
Female & 75 \\
Age, median (range) (year) & $32(18-58)$ \\
Surgical staging, no.* & \\
Stage 1 & 49 \\
Stage 2 & 39 \\
Stage 3 & 67 \\
Surgical treatment, no. & \\
Intralesion excision (curettage) & 90 \\
En bloc resection & 65 \\
Clinical course (first event), no. & \\
Disease free & 83 \\
Local recurrence & 58 \\
Metastasis & 14 \\
\hline
\end{tabular}

Disease-free survival was defined from the date of diagnosis to the event or the last day of follow-up.

${ }^{*}$ U Using the Enneking surgical staging system for GCT. ${ }^{15}$

disease free, 58 had local relapses, and 14 had lung metastasis (Table 1). Minimum follow-up for diseasefree patients was set at 60 months. The tissue of all specimens used for the study was vital or solid, without aneurysmal bone cyst-like areas.

\section{Proteomics}

\section{Two-Dimensional Gel Electrophoresis}

Proteomics analysis was performed on frozen primary tumor tissues, available from the Rizzoli Institute tissue bank, from 5 of 14 patients with GCT who subsequently developed lung metastases. The comparison was performed with primary tumor tissues from five other patients with GCT who remained continuously disease free for minimum follow-up of 5 years (Table 2). Frozen samples were homogenized with lysis buffer (8 mol/L urea, $2 \mathrm{~mol} / \mathrm{L}$ thiourea, $4 \% \mathrm{CHAPS}, 20 \mathrm{mmol} / \mathrm{L}$ dithiothreitol [DTT], 1\% Protease Inhibitor Mix [GE Healthcare, Buckinghamshire, UK], and 1\% Nuclease Mix [GE Healthcare]) and then were sonicated. After centrifugation at $13,000 \times g$ for 10 minutes, the supernatant was purified twice using the 2-D Clean-Up Kit

Table 2. Clinical Data of Patients with GCT Studied Using 2-DE and Mass Spectrometry

\begin{tabular}{|c|c|c|c|c|}
\hline Patient no./sex/age (year) & Site & Outcome* & Stage $^{+}$ & Surgery \\
\hline $1 / F / 28$ & Femur & Disease free & Active & Resection \\
\hline 2/F/22 & Tibia & Lung metastasis & Aggressive & Resection \\
\hline $3 / \mathrm{M} / 23$ & Tibia & Disease free & Active & Curettage \\
\hline $4 / F / 57$ & Sacrum & Lung metastasis & Aggressive & Resection \\
\hline $5 / F / 15$ & Femur & Disease free & Latent & Resection \\
\hline $6 / F / 28$ & Metacarpus & Lung metastasis & Aggressive & Curettage \\
\hline $7 / F / 25$ & Humerus & Disease free & Aggressive & Resection \\
\hline 8/F/23 & Radio & Lung metastasis & Aggressive & Resection \\
\hline $9 / \mathrm{M} / 36$ & Fibula & Disease free $\neq$ & Aggressive & Resection \\
\hline 10/F/17 & Femur & Lung metastasis & Active & Curettage \\
\hline
\end{tabular}

*Follow-up at 60 months.

${ }^{\dagger}$ Latent $=$ stage $1 ;$ active $=$ stage $2 ;$ and aggressive $=$ stage 3.

fLocally relapsed at 78 months. 
(GE Healthcare), and protein concentration was determined using Protein Assay based on the Bradford method (Bio-Rad Laboratories, Hercules, CA).

Protein expression profiles were obtained using twodimensional image analysis (2-DE). A total of $150 \mu \mathrm{g}$ of protein of each sample was diluted in $340 \mu \mathrm{L}$ of rehydration buffer ( $8 \mathrm{~mol} / \mathrm{L}$ urea, $2 \mathrm{~mol} / \mathrm{L}$ thiourea, 4\% CHAPS, 20 $\mathrm{mmol} / \mathrm{L}$ DTT, $1 \%$ Protease Inhibitor Mix [GE Healthcare], $0.6 \%$ IPG buffer pH3-11 [GE Healthcare], and 56\% DeStreak Rehydration Solution [GE Healthcare]) and then was applied to precast IPG strips pH3-11 (18 cm) (GE Healthcare) for isoelectric focusing. Before entering the second dimension, strips were subsequently equilibrated in two steps with equilibration buffer $(50 \mathrm{mmol} / \mathrm{L}$ Trishydrochloride [pH 8.8], $6 \mathrm{~mol} / \mathrm{L}$ urea, 30\% glycerol, and $2 \%$ SDS), adding $0.5 \%$ DTT in the first step and $4.5 \%$ iodoacetamide in the second. Twelve percent SDS-polyacrylamide gel electrophoresis cast gels were used for electrophoresis in the second dimension. Gels were stained with silver nitrate for image analysis. Three replicates of each sample were performed.

\section{Two-Dimensional Image Analysis}

2-DE gels were scanned using a GS-800 densitometer (Bio-Rad Laboratories), and the data were analyzed using Progenesis Same Spots software (Nonlinear Dynamics, Durham, NC). The silver-stained scanned images were matched, and artifacts, damaged areas, and spots on the gels or on the scanner bed were removed. 2-DE gels were grouped into metastatic and nonmetastatic samples to measure differential expression patterns between the two groups. Analysis using Progenesis software included spot detection, background subtraction, normalization, and matching. During analysis, images were organized into a match set, and alignment of member gels was produced on a reference image selected from a nonmetastatic sample. Thus, we obtained a single representative spot pattern and applied the same outline for each spot to the corresponding spot on every image in the experiment. A real measurement was obtained for each image and consistently achieved 100\% matching. Using analysis of variance univariate analysis and the $\chi^{2}$ test with the Fisher exact test, the mean quantity of each matched spot on metastatic samples was compared with the mean quantity of the corresponding spot on nonmetastatic samples; significance was set at $P<0.05$. Principal components analysis, correlation analysis, power analysis, and q-values (false discovery rate-adjusted $P$ values) were included to further explore data trends and to obtain an overview of protein spots defined as significant $(P<0.05)$ and selected to be identified by mass spectrometry.

\section{Spot Excision and Tryptic Digestion of Proteins}

Spots of interest were manually excised. Digestion was performed as previously described, ${ }^{16}$ with minor variations as follows. Silver-stained gel plugs were destained with a $1: 1$ working solution of $15 \mathrm{mmol} / \mathrm{L}$ potassium ferricyanide and $50 \mathrm{mmol} / \mathrm{L}$ sodium thiosulfate to remove the silver and then were rinsed with water and dehydrated with $100 \%$ acetonitrile. Next, plugs were treated with 10 $\mathrm{mmol} / \mathrm{L}$ DTT in $25 \mathrm{mmol} / \mathrm{L}$ ammonium bicarbonate and subsequently with $55 \mathrm{mmol} / \mathrm{L}$ iodoacetamide in 50 $\mathrm{mmol} / \mathrm{L}$ ammonium bicarbonate. Gel pieces were rinsed with $100 \%$ acetonitrile and $20 \mathrm{mmol} / \mathrm{L}$ ammonium bicarbonate. Modified porcine trypsin, at a final concentration of $6 \mathrm{ng} / \mu \mathrm{L}$ in $20 \mathrm{mmol} / \mathrm{L}$ ammonium bicarbonate (Promega Corp., Madison, WI), was added to the dry gel pieces and incubated at $37^{\circ} \mathrm{C}$ for 16 hours. Tryptic peptides were recovered and dried in a speed vacuum system and were resuspended with $0.1 \%$ trifluoroacetic acid and $50 \%$ acetonitrile in a final extraction volume of $5 \mu \mathrm{L}$.

\section{Mass Determination of Tryptic Peptides and Protein Identification}

For matrix-assisted laser desorption ionization time-offlight (MALDI-TOF) peptide mass fingerprinting, a $0.5-\mu \mathrm{L}$ aliquot of matrix solution (5 g/L of 2,5-dihydroxybenzoic acid in $33 \%$ aqueous acetonitrile plus $0.1 \%$ trifluoroacetic acid) was manually loaded onto a 400- $\mu$ m-diameter AnchorChip target plate (Bruker-Franzen Analytic $\mathrm{GmbH}$, Bremen, Germany) probe, and $1 \mu \mathrm{L}$ of the previously described peptide extraction solution was added and allowed to dry at room temperature. Samples were analyzed using a Bruker Ultraflex MALDI-TOF mass spectrometer (Bruker-Franzen Analytic $\mathrm{GmbH}$ ). The acquisition mass range was set at 600 to $4000 \mathrm{Da}$. Equipment was first externally calibrated using protonated mass signals from a standard peptide calibration mixture that contained seven peptides (Bruker Peptide Calibration Standard No. 206196; Bruker Daltonics, Billerica, MA) covering the 1000 to $3200 \mathrm{~m} / \mathrm{z}$ range, and, thereafter, every spectrum was internally calibrated using selected signals arising from trypsin autoproteolysis (842.510- and 2211.105-Da peptides) to reach a typical mass measurement accuracy of $\pm 30 \mathrm{ppm}$. Each raw spectrum was opened using FlexAnalysis 3.0 software (Bruker Daltonics), processed, and analyzed using the following variables: signal-to-noise threshold of 1, Savitzky-Golay algorithm for smoothing, tangential algorithm for baseline subtraction, and centroid algorithm for monoisotopic peak assignment. In all cases, resolution was higher than 9000. All known contaminants (trypsin autoproteolysis and known keratin peaks) were excluded during the process. Generated peaks were sent to the Mascot Server (version 2.1, May 2005) using Bio Tools 3.1 software (Bruker Daltonics) and were searched against the MSDB database (August 31, 2006; 3239079 sequences). Search variables were set as follows: searches were restricted to Homo sapiens sequences (148148 sequences), up to one missed tryptic cleavage, mass accuracy of $100 \mathrm{ppm}, \mathrm{MH}+$ monoisotopic masses, carbamidomethyl cysteine as fixed modification, and methionine oxidation as variable modification. Molecular weight search scores greater than 64 were considered significant $(P<0.05)$. 


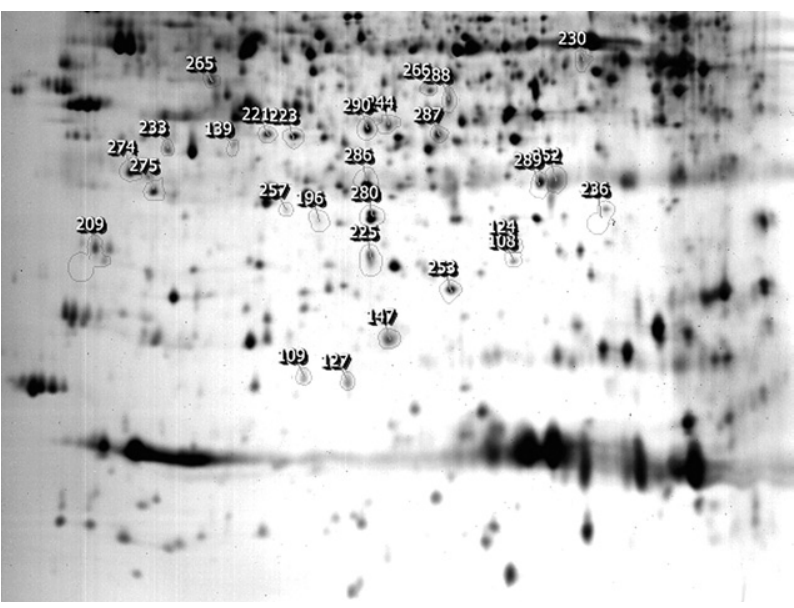

Figure 1. 2-DE reference image showing the 28 spots differentiating metastatic from nonmetastatic samples. Three replicates of every sample were performed. Statistical significance was set at $P<0.05$.

\section{Western Blotting}

Five proteins of interest were further studied by means of Western blot analysis. According to standard procedures, protein extracts from the 10 GCTs previously analyzed using 2DE were prepared by mincing and homogenizing fresh samples in extraction buffer $(50 \mathrm{mmol} / \mathrm{L}$ Tris-hydrochloride at $\mathrm{pH} 8,150 \mathrm{mmol} / \mathrm{L}$ sodium chloride, 1 $\mathrm{mmol} / \mathrm{L}$ DTT, $50 \mathrm{mmol} / \mathrm{L}$ sodium fluoride, $0.5 \%$ sodium deoxycholate, $0.1 \%$ SDS, $1 \%$ Nonidet P-40, $0.1 \mathrm{mmol} / \mathrm{L}$ PhenylMethaneSultonylFluoride, $1 \mu \mathrm{g} / \mathrm{ml}$ aprotinin, $1 \mu \mathrm{g} / \mathrm{ml}$ leupeptin, $5 \mu \mathrm{mol} / \mathrm{L}$ pepstatin, $0.1 \mathrm{mmol} / \mathrm{L}$ sodium vanadate, and $1 \mu \mathrm{mol} / \mathrm{L}$ microcystin). Protein extracts (50 $\mu \mathrm{g}$ ) were analyzed by means of $12 \%$ SDS-polyacrylamide gel electrophoresis, and Western blot was performed by using anti-glutathione peroxidase 1 (GPX1) (Abcam, Cambridge, UK) diluted 1:1000, anti-thioredoxin peroxidase (PRX) (Santa Cruz Biotechnology, Santa Cruz, CA) diluted 1:200, anti-allograft inflammatory factor 1 (AIF1) (Santa Cruz Biotechnology) diluted 1:25, anti-ubiquitin E2N (UBE2N) (Invitrogen, Camarillo, CA) diluted 1:100, and anti-heat shock protein 27 (HSP27) (Santa Cruz Biotechnology) diluted 1:100. The signal was visualized using SuperSignal West Pico Chemiluminescent Substrate (Pierce, Rockford, IL) and was quantified by densitometric analysis using Quantity One software (Bio-Rad Laboratories). Liver mouse lysate, K-562 whole cell lysate, rat brain extract, HepG2 cell lysate, and Hela cell lysate were respectively used as positive controls.

\section{Tissue Microarray and Immunohistochemical Analysis}

Tissue microarray (TMA) sections were used to validate protein expression on a series of 164 primary GCT lesions from biopsy or surgical specimens of patients

Table 3. Differentially Expressed Spots by 2-DE

\begin{tabular}{|c|c|c|c|c|c|}
\hline \multirow[b]{2}{*}{ Rank } & \multirow[b]{2}{*}{ ANOVA ( $P$ value) } & \multirow[b]{2}{*}{ Fold } & \multirow[b]{2}{*}{ Notes } & \multicolumn{2}{|c|}{ Average normalized volumes* } \\
\hline & & & & Metastatic & Nonmetastatic \\
\hline 108 & 0.002 & 3.6 & ID: 0001 & $33,429.766$ & 9230.593 \\
\hline 109 & $2.666 \times 10^{-4}$ & 3.6 & ID: 0742 & $24,536.878$ & 6780.591 \\
\hline 124 & $2.371 \times 10^{-4}$ & 3.0 & ID: 0594 & $48,529.908$ & $15,929.501$ \\
\hline 127 & 0.007 & 3.1 & ID: 0745 & $22,000.687$ & 7100.310 \\
\hline 139 & 0.010 & 2.9 & ID: 0474 & 7747.085 & $22,094.187$ \\
\hline 147 & $5.025 \times 10^{-4}$ & 2.6 & ID: 0706 & $64,969.293$ & $24,721.118$ \\
\hline 196 & 0.006 & 2.1 & ID: 0560 & $25,802.943$ & $53,538.373$ \\
\hline 209 & $1.965 \times 10^{-4}$ & 2.0 & ID: 0012 & $84,783.299$ & $169,283.981$ \\
\hline 221 & $4.530 \times 10^{-5}$ & 1.9 & ID: 0446 & $30,302.595$ & $59,005.451$ \\
\hline 223 & $4.414 \times 10^{-5}$ & 1.9 & ID: 0452 & $43,725.789$ & $84,764.152$ \\
\hline 225 & 0.003 & 1.9 & ID: 0603 & $167,166.958$ & $86,531.382$ \\
\hline 230 & 0.002 & 1.9 & ID: 0303 & $50,510.763$ & $26,681.587$ \\
\hline 233 & 0.010 & 1.9 & ID: 0472 & $23,291.267$ & $12,552.152$ \\
\hline 236 & 0.014 & 1.8 & ID: 0010 & $41,562.188$ & $76,220.855$ \\
\hline 244 & $9.056 \times 10^{-4}$ & 1.8 & ID: 0432 & $39,892.330$ & $70,362.549$ \\
\hline 252 & 0.004 & 1.7 & ID: 0509 & $84,068.223$ & $48,877.373$ \\
\hline 253 & 0.019 & 1.7 & ID: 0652 & $51,498.321$ & $88,068.599$ \\
\hline 257 & 0.005 & 1.7 & ID: 0552 & $12,364.124$ & $20,451.643$ \\
\hline 265 & 0.009 & 1.6 & ID: 0349 & $33,255.640$ & $21,329.063$ \\
\hline 266 & 0.010 & 1.6 & ID: 0363 & $21,003.306$ & $32,734.419$ \\
\hline 274 & 0.018 & 1.5 & ID: 0496 & $75,487.099$ & $115,419.807$ \\
\hline 275 & 0.026 & 1.5 & ID: 0528 & $63,855.276$ & $95,949.379$ \\
\hline 280 & 0.016 & 1.4 & ID: 0554 & $100,391.376$ & $145,364.060$ \\
\hline 286 & $5.842 \times 10^{-4}$ & 1.4 & ID: 0512 & $97,721.339$ & $132,228.870$ \\
\hline 287 & 0.028 & 1.3 & ID: 0445 & $34,724.958$ & $46,859.401$ \\
\hline 288 & 0.007 & 1.3 & ID: 0374 & $34,703.750$ & $45,602.258$ \\
\hline 289 & 0.004 & 1.2 & ID: 0518 & $69,137.967$ & $86,296.423$ \\
\hline 290 & 0.026 & 1.2 & ID: 0438 & $63,418.691$ & $75,268.495$ \\
\hline
\end{tabular}

ANOVA, analysis of variance.

${ }^{*}$ Calculated using Progenesis Same Spot software. The intensity of each protein spot was normalized as a percentage of total volume, corresponding to pixel intensity integrated over the area of each spot and divided by the sum of all spots in the gel to account for staining variability. Average normalized spot volumes (pixel intensity over spot area) were compared between metastatic and nonmetastatic tumors. 
A

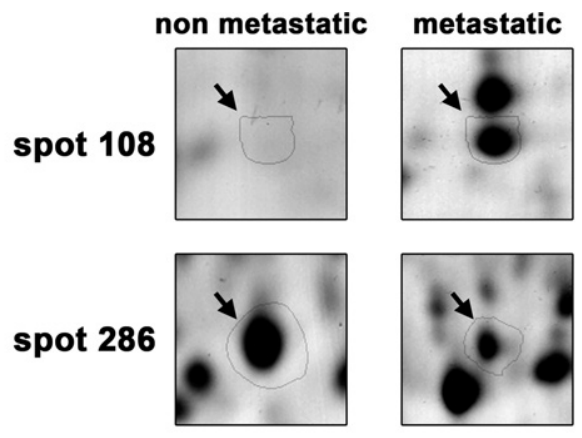

B

levels throughout gels
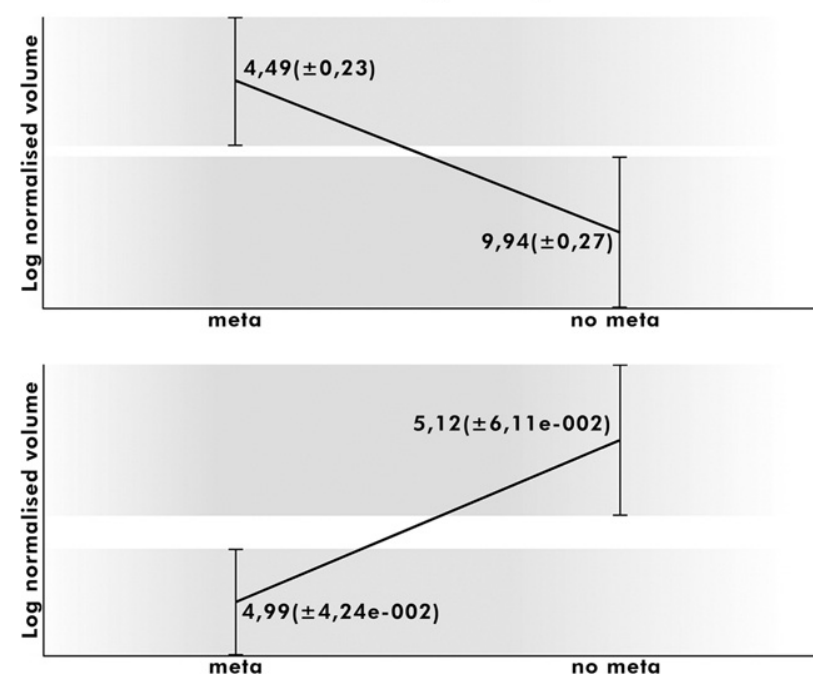

Figure 2. Representative images from Progenesis Same Spot software analysis. A: Arrows indicate the position of differentially expressed spots (108 and 286) in proteomic maps from metastatic and non metastatic GCT. B: The lines show expression levels of spots SSP 108 and 286 throughout the gels.

included in 198 blocks to be fixed in 10\% formalin, processed, and paraffin embedded. H\&E-stained slides of all the samples were reviewed by the pathologist (T.K.) to choose representative areas for TMA construction at the Semmelweis University of Budapest by using the computer-driven Mirax TMA system (manufactured by 3DHistech Ltd., Budapest, Hungary, and distributed by Zeiss Jena GmbH, Jena, Germany). The Excel spreadsheets (Microsoft Corp, Redmond, WA) containing clinical and histologic patient data, reviewed by the pathologist (P.P.), were imported by the software to be linked to relevant TMA core positions during TMA building. Tissue cores measuring $2 \mathrm{~mm}$ in diameter were placed in three pieces of 80-sample TMA blocks forming 240 samples, of which some were repeated samples from the same patient. Serial 4- $\mu$ m-thick TMA sections were used for immunohistochemical analysis. Excluding lost or damaged samples, expression data for GPX1, PRX, AIF1, UBE2N, and HSP27 proteins were finally available from 155 primary GCTs (Table 1) after immunostaining. According to the manufacturer's protocol, we used rabbit polyclonal anti-GPX1 diluted 1:300 (Abcam), rabbit polyclonal antiPRX diluted 1:25 (Santa Cruz Biotechnology), mouse monoclonal anti-AIF1 diluted 1:50 (Santa Cruz Biotechnology), mouse monoclonal anti-UBE2N diluted 1:100
(Invitrogen), and mouse monoclonal anti-HSP27 diluted 1:100 (Santa Cruz Biotechnology) as primary antibodies. Streptavidin-biotin peroxidase DAB rabbit/mouse (Dako, Glostrup, Denmark) was used as a detection system. No antigen retrieval methods were needed. Staining results were interpreted by the pathologist (P.P.). According to the percentage of positive tumor cells, samples were scored as negative (no positive cells), weakly positive ( $<25 \%$ positive cells), moderately positive ( $25 \%$ to $49 \%$ positive cells), or strongly positive ( $\geq 50 \%$ positive cells). Protein overexpression was considered only in the presence of moderate to strong positivity, corresponding to a staining of more than $25 \%$ of tumor cells. Human kidney (GPX1), breast (PRX), brain (AIF1 and UBE2N), and breast carcinoma (HSP27) tissue sections were used as

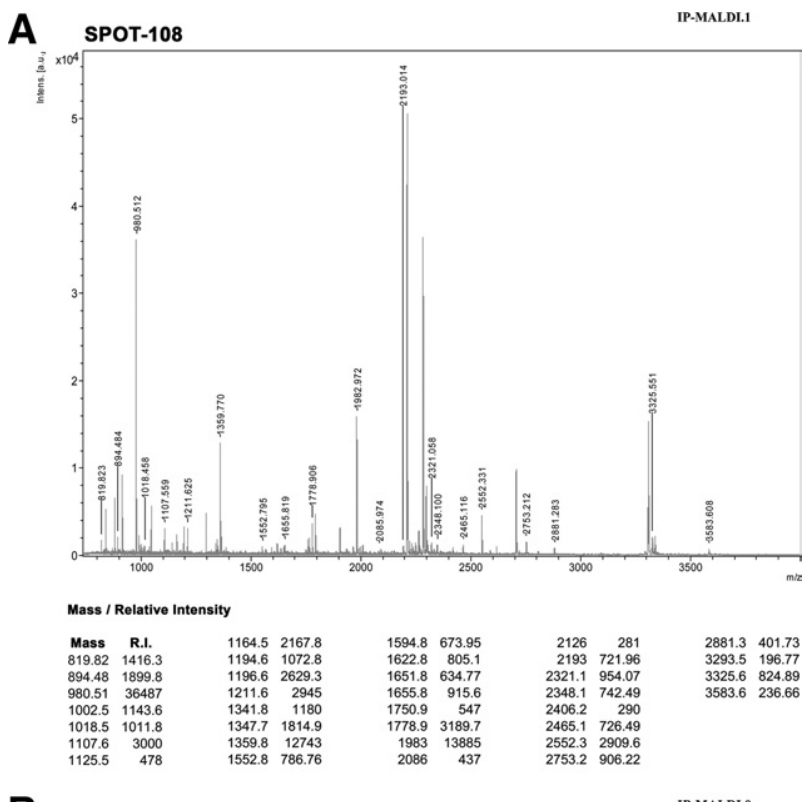
B
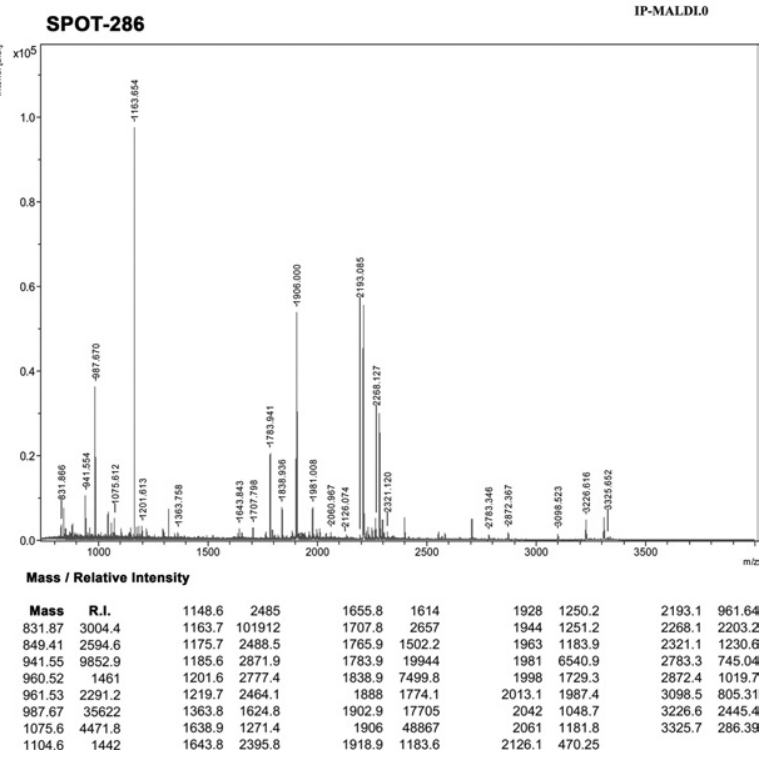

Figure 3. Representative mass spectrometry scan of tryptic peptides of spots 108 (A) and 286 (B) identified as PRX and HSP27, respectively. Mass value $(\mathrm{m} / \mathrm{z})$ and putative amino acid position assignments are indicated above the peaks. Assignments were made using Mascot software. Mass value and relative intensity are reported for each peptide. 
Table 4. Identified Protein Functional Categories

\begin{tabular}{|c|c|c|c|c|c|}
\hline Rank & Candidate proteins & Accession no. & Functional categories & Fold & $\begin{array}{c}\text { Alterations in metastatic } \\
\text { tumors }\end{array}$ \\
\hline 108 & $\operatorname{PRX}$ & A46711 & Antioxidant enzymes & 3.6 & Overexpression \\
\hline 109 & AIF1 & JC5246 & Inflammatory response protein & 3.6 & Overexpression \\
\hline 127 & UBE2N & JC4894 & Ubiquitination protein & 3.1 & Overexpression \\
\hline 147 & Cofilin 1 & COF1 & Cytoskeletal protein & 2.6 & Overexpression \\
\hline 209 & Lysozyme mutant $\mathrm{T} 11 \mathrm{~A}$ & $1 \mathrm{CJ} 6 \mathrm{~A}$ & Hydrolase & 2.0 & Underexpression \\
\hline 221 & NADH dehydrogenase (ubiquinone) & JE0195 & Oxidoreductase & 1.9 & Underexpression \\
\hline 223 & Thiol-specific reductase & GO1790 & Oxidoreductase & 1.9 & Underexpression \\
\hline 225 & GPX1 & OPHUE & Antioxidant enzymes & 1.9 & Overexpression \\
\hline 230 & GAPDH & G3P & Oxidoreductase & 1.9 & Overexpression \\
\hline 233 & Apolipoprotein A1 protein (fragment) & CAA00975 & Lipoprotein & 1.9 & Overexpression \\
\hline 244 & $\begin{array}{l}\text { Dodecenoyl-CoA delta-isomerase } \\
\text { precursor }\end{array}$ & A55723 & Metabolic protein & 1.8 & Underexpression \\
\hline 253 & Cdc42 mutant Q61L, chain A & $1 \mathrm{CF} 4 \mathrm{~A}$ & GTPase & 1.7 & Underexpression \\
\hline 257 & PSMB4 proteasome subunit & Q6IBI4 & Proteasome protein & 1.7 & Underexpression \\
\hline 286 & HSP27 & HHHU27 & Heat shock protein & 1.4 & Underexpression \\
\hline
\end{tabular}

GAPDH, glyceraldehyde-3-phosphate dehydrogenase; PSMB4, proteasome (prosome, macropain) subunit, beta type, 4.

positive controls. Negative controls were performed omitting the primary antibody.

\section{Statistical Analysis}

The $\chi^{2}$ test with the Fisher exact test was used to establish the association between prognosis and protein expression levels. Disease-free survival was calculated using Kaplan-Meier analysis, and the curve comparison was performed using the Breslow test.

\section{Results}

\section{Identification of Differentially Expressed Proteins}

Silver-stained 2-DE gels performed on five metastatic and five nonmetastatic GCT protein lysates showed an average of 300 spots. Image analysis using Progenesis Same Spot software allowed us to identify 28 spots differentially expressed between primary metastatic and nonmetastatic GCTs (Figure 1). Spots were ranked by $P$ value from the one-way analysis of variance, defining the value of 0.05 as a significant threshold, and were given a unique ID (Table 3). Analyses used to explore the trends included principal components analysis, correlation analysis, power analysis, and q-values. These, using a range of displays, provided a statistically ordered list, where in the metastatic group 10 were up-regulated and 18 were down-regulated (Table 3; Figure 2). After validating 2-DE with another independent 2-DE, 19 of these 28 spots were subsequently chosen to be analyzed by mass spectrometry using MALDI-TOF. Eighteen spots were selected according to fold value, and an additional spot was chosen for its very low $P$ value $(P=5.842 \mathrm{e}-004)$ (Table 3). After excision and gel tryptic digestion, extracted peptides were analyzed using MALDI-TOF (Figure 3). Five did not enter the criteria for MALDI-TOF analysis, and 14 spots were identified by the MSDB database. The levels of seven of these proteins were increased and seven were decreased in metastatic compared with disease-free samples, and their functional categories are reported in Table 4, showing that PRX, AIF1, and UBE2N had more than threefold overexpression in metastatic compared with nonmetastatic tumors. The fold factor for the decreased proteins ranged from 1.4-fold for HSP27 to two-fold for the lysozyme mutant T11A (Table 4).

\section{Expression Levels and Validation of Selected Proteins}

On the basis of these data, we selected proteins involved in cell cycle or apoptosis. Moreover, PRX, AlF1, and UBE2N had more than threefold up-regulation; GPX1 had 1.9-fold up-regulation but was associated to redox signaling, similar to PRX; and HSP27 showed down-regulation in metastatic samples with a very low $P$ value. By Western blot analysis, tumor samples revealed de novo or higher amounts of GPX1, PRX, and AlF1 proteins with respect to the paired normal tissues unless expression was not detectable (Figure 4). When we compared metastatic and nonmetastatic samples, we found that the differences in GPX1 and AIF1 were consistent with 2-DE data, showing weak or undetectable expression in all nonmetastatic samples except case 9, which had a late local relapse (Figure 4; Table 5). Although variability in UBE2N expression was observed, evaluation of the electrophoretic band volume demonstrated a trend toward increased expression in metastatic samples, whereas PRX and HSP27 proteins were resolved without marked differences between metastatic and nonmetastatic groups (Figure 4; Table 5). In three cases, HSP27 was expressed more in normal tissue. Case 9, which had a late local relapse, showed strong expression of all the proteins studied.

\section{Histologic Features of Human GCT Tissue Sections}

All tissue sections had high cellular density, with many multinucleated osteoclast-like cells derived from monocyte-macrophage lineage and a mononuclear compo- 


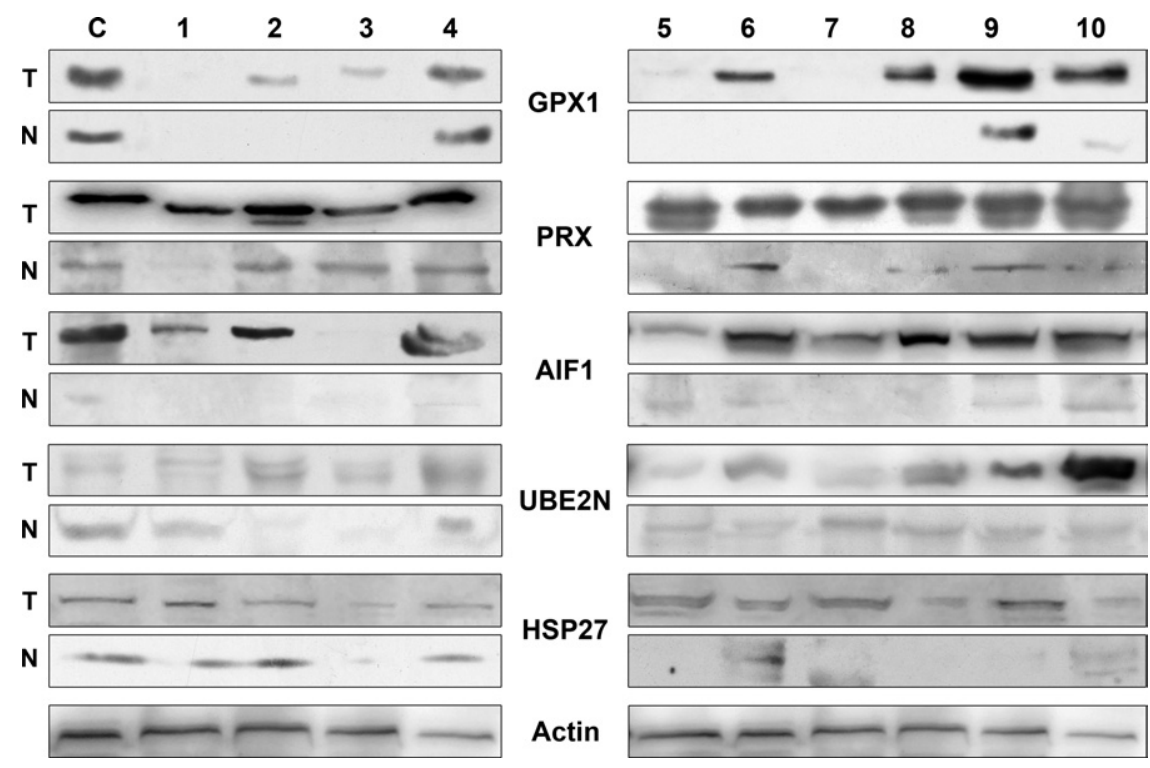

Figure 4. Western blot analysis using Quantity One software shows changes of protein amount in GCTs compared with normal paired tissues. Lines $1,3,5,7$, and 9 correspond to nonmetastatic samples. Lines 2, 4, 6, 8, and 10 correspond to metastatic samples. Case 9 had a late loca relapse at 78 months. GPX1 and AIF1 have weak or undetectable expression in nonmetastatic samples, except in case 9. UBE2N demonstrated a trend toward increased expression in metastatic samples, whereas PRX and HSP27 proteins were resolved without significant differences between the two groups. Actin was used as a reference protein. C, control; T, tumor; $N$, paired normal tissue. nent consisting of spindle-shaped fibroblast-like cells and round macrophage-like cells (Figure 5).

\section{Immunohistochemical Detection and Clinical Correlation}

Immunostaining performed on TMA slides of 155 paraffinembedded sections of primary GCTs with different clinical outcomes including locally relapsed, metastatic, and disease-free patients with minimum follow-up of 60 months revealed a higher percentage of overexpression in the locally relapsed group compared with the diseasefree group for GPX1 (71\% versus $24 \%, \chi^{2}=35.04, P=$ $0.0001)$, PRX ( $65 \%$ versus $54 \%, P=\mathrm{NS})$, and $\operatorname{AlF} 1$ ( $63 \%$ versus $36 \%, \chi^{2}=15.0, P=0.03$ ). Significant differences in GPX1, PRX, and AlF1 overexpression were also seen between the disease-free and metastatic groups, where most samples showed immunoreactivity in more than $50 \%$ of cells for each, with variable cytoplasm stain intensity and distribution (Table 6). In detail, locally relapsed and metastatic GCTs showed strong and diffused GPX1, PRX, and AIF1 immunostaining in multinuclear cells ( $\geq 50 \%$ positive cells). In the mononuclear component, PRX was uniformly positive ( $\geq 50 \%$ positive cells), whereas GPX1 and AIF1 were focally expressed (25\% to $49 \%$ positive cells). All relapsed samples with strong expression of PRX also had increased expression of
GPX1. In most tumors from disease-free patients, the detection of these proteins was weak $(<25 \%)$ or negative in mononuclear cells, whereas multinucleated cells were less strongly stained than were locally relapsed and metastatic tumors (Figure 5). Comparing the frequency of overexpression, UBE2N had a higher percentage of cases in the locally relapsed and metastatic groups than in the disease-free group (52\% versus $42 \%, P=N S$ and $86 \%$ versus $42 \%, P=0.05$, respectively) (Table 6 ). In locally relapsed and metastatic GCTs, staining was strongly diffused on the membranes of mononuclear cells ( $\geq 50 \%$ positive cells), whereas multinucleated cells were predominantly negative. In the disease-free group, UBE2N was often undetectable (Figure 5). No significant differences were seen for HSP27 in terms of overexpression frequency (Table 6). HSP27 showed positive staining in the mononuclear cell cytoplasm with variable intensity and distribution (Figure 5)

When the clinical course of patients who presented with primary lesions with GPX1 overexpression corresponding to moderate to strong positivity ( $\geq 25 \%$ positive cells) was matched with that of patients with weak or undetectable expression ( $<25 \%$ positive cells), the latter were found to have an estimated disease-free survival of $79 \%$ versus $26 \%$ for patients with moderate to strong immunoreactivity. Accordingly, the difference between Kaplan-Meier curves was significant (Breslow test $=$

Table 5. Adjusted Volume Quantified by Densitometric Analysis Software

\begin{tabular}{|c|c|c|c|c|c|c|c|c|c|c|}
\hline \multirow[b]{2}{*}{ Variables } & \multicolumn{10}{|c|}{ Samples* } \\
\hline & 1 & 2 & 3 & 4 & 5 & 6 & 7 & 8 & 9 & 10 \\
\hline GPX1 & 0.05 & 2.2 & 2.0 & 6.6 & 1.2 & 8.0 & 0.05 & 7.4 & 15.2 & 10.8 \\
\hline PRX & 5.0 & 13.5 & 6.9 & 11.9 & 7.7 & 8.96 & 7.63 & 10.53 & 9.46 & 11.42 \\
\hline AlF1 & 2.1 & 13.4 & 0.05 & 9.2 & 2.0 & 10.0 & 5.0 & 11.5 & 14.4 & 12.1 \\
\hline UBE2N & 0.8 & 2.7 & 1.9 & 3.3 & 1.0 & 4.3 & 0.8 & 7.0 & 9.5 & 40.4 \\
\hline HSP27 & 4.43 & 2.9 & 2.78 & 3.15 & 7.13 & 6.72 & 10.1 & 10.07 & 9.12 & 4.27 \\
\hline
\end{tabular}

${ }^{*}$ Samples 1, 3, 5, 7, and 9 are nonmetastatic and samples number 2, 4, 6, 8, and 10 are metastatic. Case 9 locally relapsed at 78 months. 


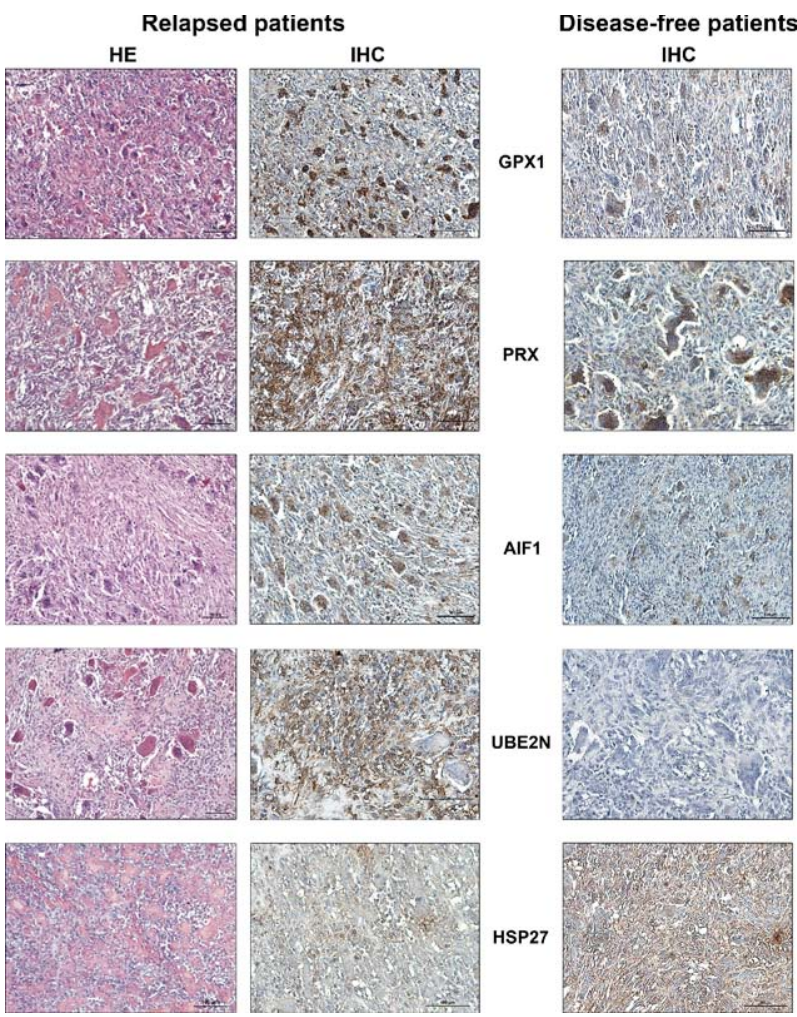

Figure 5. Left column shows hematoxylin eosin stained spots from TMA in patients who relapsed. Center column shows hematoxylin eosin stained spots immunohistochemical analysis of the same spots for the five proteins selected. Right column shows immunohistochemical analysis from patients without documented relapses at minimum disease-free follow-up of 60 months. In most tumors from disease-free patients, the detection of GPX1, PRX, and AIF1 proteins was weak or negative in mononuclear cells, whereas multinucleated cells were less strongly stained than were relapsed tumors. UBE2N is uniformly expressed on the membrane of mononuclear cells of relapsed GCT, whereas in the disease-free group, UBE2N was often undetectable. HSP27 shows positive staining in mononuclear cell cytoplasm, with variable intensity and distribution.

13.5; $P=0.0002)$, even when a cutoff point of $50 \%$ was used (Figure 6).

\section{Discussion}

Global expression profile analysis may provide important information for understanding tumor biology and discovering patterns of biomarkers associated with local and systemic relapse. This allows early identification of highrisk patients for whom more appropriate treatment regimens should be considered. ${ }^{17}$ Previous studies examined quantitative differences in protein expression in a

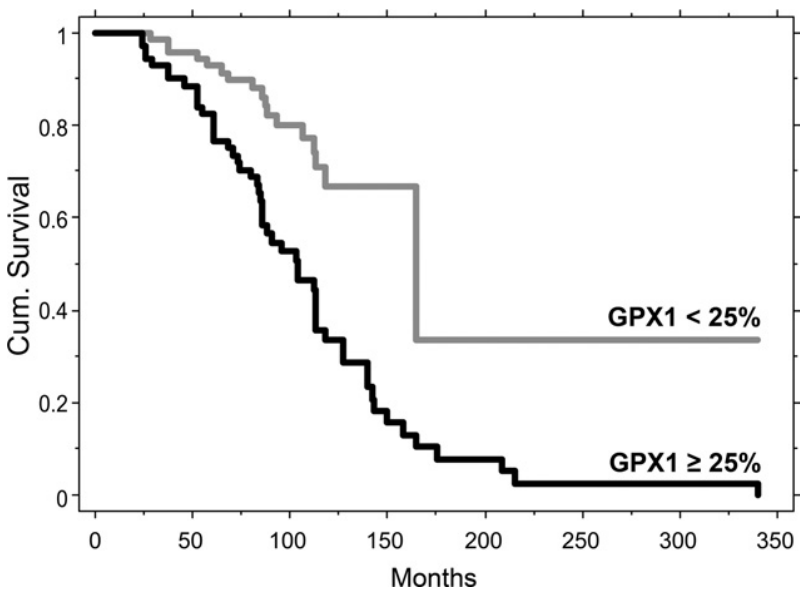

Figure 6. Kaplan-Meier curve showing patient disease-free survival in relation to GPX1 immunostaining. Patients with negative or weakly positive GPX1 expression had a higher probability of disease-free survival in terms of local recurrences or metastases at minimum follow-up of 60 months (Breslow test $=13.5 ; P=0.0002$ ).

large series of lung adenocarcinoma and identified candidate prognostic proteins associated with clinicopathologic features of these tumors. ${ }^{18}$ A cluster analysis based on 52 protein spots differentiated endometrial carcinoma from normal endometrium and showed significant differences in protein expression between carcinoma of low and high malignancy potential. ${ }^{19}$

In this study of GCTs with different clinical behavior in terms of lung metastasis occurrence, we recognized a set of 28 protein spots that differentiated metastatic from nonmetastatic patients; among them, the 14 most representative spots were selected and identified using MALDI-TOF mass spectrometry on the basis of peptide mass matching. Seven of these protein spots showed up to 3.6-fold increased expression in the metastatic group, and seven were slightly down-regulated, with an up to twofold differential expression.

Volume analysis of the electrophoretic bands corresponding to the five most representative proteins involved in cell cycle regulation and apoptosis are in line with 2-DE data, also showing, when expressed, differences between tumor and paired normal samples. Immunostaining performed on a much wider cohort of primary GCT archival specimens from patients who developed metastases or local recurrences or patients without documented relapses at minimum follow-up of 60 months detected a higher frequency of GPX1, PRX, AIF1, and UBE2N overexpression in the group of primary GCT that developed lung metastases

Table 6. Distribution of Protein Overexpression in GCT Subgroups

\begin{tabular}{cccccc}
\hline Variables & $\begin{array}{c}\text { Locally relapsed, no. }(\%) \\
(n=58)\end{array}$ & $\begin{array}{c}\text { Disease free, no. }(\%) \\
(n=83)\end{array}$ & $\chi^{2}(P$ value $)$ & $\begin{array}{c}\text { Metastatic, no. }(\%) \\
(n=14)\end{array}$ & $\chi^{2}(P$ value $)$ \\
\hline GPX1 & $41(71)$ & $20(24)$ & 0.0001 & $14(100)$ & 0.004 \\
PRX & $38(65)$ & $45(54)$ & NS & $11(79)$ & 0.02 \\
AlF1 & $37(64)$ & $30(36)$ & 0.03 & $14(100)$ & 0.02 \\
UBE2N & $30(52)$ & $35(42)$ & NS & $12(86)$ & 0.05 \\
HSP27 & $23(40)$ & $45(54)$ & NS & $8(57)$ & 0.07 \\
\hline
\end{tabular}

NS, not significant. 
or relapsed locally than in the disease-free group where weak and focal or absent immunoreactivity was seen in a high percentage of cases.

PRX and GPX1, closely correlated in terms of co-overexpression, are antioxidant enzymes transcriptionally induced by p53 and involved in redox signaling. They negatively control the production of reactive oxygen species after oxidative stress ${ }^{20,21}$ and protect cells against DNA damaging insults. In advanced tumors, reactive oxygen species is often down-regulated, and it has been reported that p53 can drive expression of antioxidant genes, which, in turn, decrease reactive oxygen species levels and suppress apoptosis, also affecting the clinical effects of anticancer drugs. ${ }^{22,23}$ Thus, the inhibition of apoptosis by antioxidants may exert a cancer-promoting effect in patients with cancer, and this is supported by the evidence that dietary antioxidant depletion inhibited tumor growth and diminished metastasis in transgenic mice. ${ }^{24}$

Mammalian thioredoxins are a family of small redox proteins that stimulate cell growth and inhibit apoptosis and enhance tumor angiogenesis. ${ }^{25,26} \mathrm{PRX} 1$ was found overexpressed in breast cancer, and its mRNA levels were associated with tumor grade, ${ }^{27}$ and PRX4 was recently found to be overexpressed in osteosarcoma cell lines and tissues compared with in osteoblast cells used as control. ${ }^{28} \mathrm{GPX} 1$, a direct p53 antiapoptotic target, inhibits oxidative stressinduced apoptosis, increasing anticancer drug resistance by p53 inactivation. ${ }^{29-31}$ In our series of wild-type p53 GCTs, the significant association between high levels of GPX1 and the occurrence of local relapse or metastasis may indicate an imbalance in reactive oxygen speciesactivated signaling pathways, resulting in negative control of p53 that leads to cell survival. This antiapoptotic effect could be reinforced by the diffused and strong expression of PRX1 and the presence of UBE2N on the membrane of mononuclear cells in GCTs, promoting p53 nuclear export with consequent reduction of its transcriptional activity and ability to induce apoptosis. ${ }^{32}$

AIF1, strongly and uniformly expressed in multinuclear cells in relapsed GCTs, is involved in inflammatory response $^{33}$ and is implicated in angiogenesis, proliferation, and development of tumors through endothelial cell activation, signal transduction, and vasculogenesis. ${ }^{34,35}$ Some data reported the role of AIF1 in breast tumor growth and proliferation via activation of nuclear factor kappa B signaling ${ }^{36}$ In vitro studies demonstrated that up-regulation of AlF1 expression may increase transcriptional activity of nuclear factor kappa B, which, in turn, affects the expression of cyclin D1 and accelerates cell entry into S-phase. ${ }^{36-38}$

In accordance with these results, some studies demonstrated a tight association between inflammatory cytokines and malignant progression. ${ }^{39,40}$ Our previous data ${ }^{41}$ pointed out that during bone resorption, GCTs express growth factors and cytokines that, in turn, enhance AIF1, crucial for survival and pro-inflammatory activity of macrophages. ${ }^{42}$ This may represent a link between osteoclast activity and inflammation.

Among the down-regulated spots, we identified HSP27, whose expression has been found to be functionally linked to apoptosis and drug resistance in different tumors. ${ }^{43,44} \mathrm{HSPs}$ are highly conserved stress proteins induced by a variety of insults, such as hyperthermia and oxidative stress, ${ }^{45,46}$ and their activation allows cells to survive and increases the tumorigenic potential of cancer cells. ${ }^{47}$ In our series, HSP27 had great variability in staining intensity and distribution, and no significant differences were found between locally relapsed, metastatic, and disease-free GCTs.

Finally, when we compared patient survival in relation to immunostaining intensity and distribution of the previously identified proteins, Kaplan-Meier analysis revealed that patients with GPX1 overexpression had a higher probability of relapses, including local recurrences and lung metastases, than did patients with weak or undetectable expression. This suggests that up-regulation of GPX1 may identify a subset of high-risk patients with GCT prone to receive diverse clinical management.

However, other important proteins that could be useful for GCT patient stratification have been found differently expressed by 2-DE, including cofilin, whose activity is required for tumor cell motility and invasion ${ }^{48}$; dodecenoyl-CoA delta-isomerase, a biomarker for early diagnosis and therapeutic monitoring of breast disease ${ }^{49}$; and $\mathrm{CDC} 42$, a key regulator of cell migration, adhesion, and actin dynamics. ${ }^{50}$

In conclusion, our data confirm that despite the rarity of GCT, the use of proteomics technologies associated with the clinicopathologic characteristics of patients provides important results by revealing a list of antiapoptotic or proliferating biomarkers that may be useful in predicting their clinical course. The importance of this study is to have identified new proteins involved in tumor cell survival and associated with an increased aggressiveness of GCT that leads, in most cases, to local recurrences and, more rarely, to metastasis. Additional studies relative to the enzymatic and biological activity of these molecules are needed to provide further information on signaling pathways involved in GCT development and local or systemic relapses.

\section{Acknowledgments}

We thank Ms. Edit Parsch for her technical assistance in creating the TMA, Dr. Alba Balladelli for editing the paper, Ms. Cristina Ghinelli for the graphics, and the Rizzoli Institute surgical pathology technicians.

\section{References}

1. Campanacci M: Giant cell tumor of bone. Curr Orthop 1993, 7:26-35

2. Lau YS, Sabokbar A, Gibbone CL, Giele H, Athanasou N: Phenotypic and molecular studies of giant cell tumors of bone and soft tissue. Hum Pathol 2005, 36:945-954

3. Werner M: Giant cell tumor of bone: morphological, biological and histogenetical aspects. Int Orthop 2006, 30:484-489

4. Fujisaki K, Tanabe N, Suzuki N, Kawato T, Takeiki O, Tsuzukibashi O, Makimura M, Ito K, Maeno M: Receptor activator of NF-kB lingand induces the expression of carbonic anhydrase II cathepsin $\mathrm{K}$ and metallo proteinase 9 in osteoclast percursor RAW264.7 cells. Life Sci 2007, 80:1311-1318

5. Chang SS, Suratwala SJ, Jung KM, Doppelt JD, Zhang HZ, Blaine TA, Kim TW, Winchester RJ, Lee FY: Bisphosphonates may reduce re- 
currence in giant cell tumor by inducing apoptosis. Clin Orthop 2004 1:103-109

6. Anderson P, Kopp L, Anderson N, Cornelius K, Herzog C, Hughes D, Huh W: Novel bone cancer drugs: investigational agents and control paradigms for primary bone sarcomas (Ewing's sarcoma and osteosarcoma). Expert Opin Investig Drugs 2008, 17:1703-1715

7. Sawyer JR, Goosen LS, Binz RL, Swanson CM, Nicholas RW: Evidence for telomeric fusion as a mechanism for recurring structural aberrations of chromosome 11 in giant cell tumor of bone. Cancer Genet Cytogenet 2005, 159:32-36

8. Smith LT, Mayerson J, Nowak NJ, Suster D, Mohammed N, Long S Auer H, Jones S, McKeegan C, Young G, Bos G, Plass C, Morrison C: 20q11.1 amplification in giant-cell tumor of bone: array CGH, FISH, and association with outcome. Genes Chromosomes Cancer 2006, 45:957-966

9. Wulfkuhle J, Espina V, Liotta L, Petricoin E: Genomic and proteomic technologies for individualization and improvement of cancer treatment. Eur J Cancer 2004, 40:2623-2632

10. Guenther R, Krenn V, Morawietz L, Dankof A, Melcher I, Schaser KD Kasper HU, Kuban RJ, Ungethüm U, Sers C: Giant cell tumors of bone: molecular profiling and expression analysis of Ephrin A receptor, Claudin 7, CD52, FGFR3 and AMFR. Pathol Res Pract 2005, 201:649-663

11. Skubitz KM, Cheng EY, Clohisy DR, Thompson RC, Skubitz AP: Gene expression in giant cell tumors. J Lab Clin Med 2004, 144:193-200

12. Wuelling M, Delling G, Kaiser E: Differential gene expression in stromal cells of human giant cell tumor of bone. Virchows Arch 2004 445:621-630

13. Gamberi G, Benassi MS, Ragazzini P, Pazzaglia L, Ponticelli F, Ferrari C, Balladelli A, Mercuri M, Gigli M, Bretoni F, Picci P: Proteases and interleukin-6 gene analysis in 92 giant cell tumors of bone. Ann Oncol 2004, 15:498-503

14. Kawai A, Kondo T, Suehara Y, Kikuta K, Hirohashi S: Global proteinexpression analysis of bone and soft tissue sarcomas. Clin Orthop Relat Res 2008, 466:2099-2106

15. Wolf RE, Enneking WF: The staging and surgery of musculoskeletal neoplasms. Orthop Clin North Am 1996, 27:473-481

16. Shevchenko A, Wilm M, Vorm O, Mann M: Mass spectrometric sequencing of proteins silver-stained polyacrylamide gels. Anal Chem 1996, 68:850-858

17. Espina V, Dettloff KA, Cowherd S, Petricoin EF III, Liotta LA: Use of proteomic analysis to monitor responses to biological therapies. Expert Opin Biol Ther 2004, 4:83-93

18. Chen C, Gharib TG, Huang CC, Thomas DG, Shedden KA, Taylor JM, Kardia SL, Misek DE, Giordano TJ, lannettoni MD, Orringer MB, Hanash SM, Beer DG: Proteomic analysis of lung adenocarcinoma identification of highly expressed set of proteins in tumors. Clin Cancer Res 2002, 8:2298-2305

19. Lundgren C, Lahmann J, Becker S, Roblick U, Schedvins k, Boman k, Frankendal B, Nordström B, Auer G: 2-DE protein expression in endometrial carcinoma. Acta Oncol 2006, 45:685-694

20. Ueda S, Masutani H, Nakamura H, Tanaka T, Ueno M, Yodoi J: Redox control of cell death. Antioxid Redox Signal 2002, 4:405-414

21. Mori K, Shibanuma M, Nose K: Invasive potential induced long-term oxidative stress in mammary epithelial cells. Cancer Res 2004, 64: 7464-7472

22. Sablina AA, Budanov AV, Ilyinskaya GV, Agapova LS, Kravchenko JE, Chumakov PM: The antioxidant function of p53 tumor suppressor. Nat Med 2005, 11:1306-1313

23. Gouazè V, Mirault ME, Carpentier S, Salvayre R, Levade T, AndrieuAbadie N: Glutathione peroxidase-1 overexpression prevents ceramide production and partially inhibits apoptosis in doxorubicintreated human breast carcinoma cells. Mol Pharmacol 2001, 60: $488-496$

24. Liu X, Zeng B, Ma J, Wan C: Comparative proteomic analysis of osteosarcoma cell and human primary cultured osteoblastic cell. Cancer Invest 2009, 27:345-352

25. Powis G, Montfort WR: Properties and biological activities of thioredoxins. Annu Rev Biophys Biomol Struct 2001, 30:421-455

26. Welsh SJ, Bellamy WT, Briehl MM, Powis G: The redox protein thioredoxin-1 (Trx-1) increases hypoxia-inducible factor 1alpha protein expression: trx-1 overexpression results in increased vascular endo- thelial growth factor production and enhanced tumor angiogenesis. Cancer Res 2002, 62:5089-5095

27. Cha MK, Suh $\mathrm{KH}$, Kim IH: Overexpression of peroxiredoxin I and thioredoxin 1 in human breast carcinoma. J Exp Clin Cancer Res 2009, 28:93-104

28. Albright CD, Salganik RI, Van Dyke T: Dietary depletion of vitamin E and vitamin A inhibits mammary tumor growth and metastasis in transgenic mice. J Nutr 2004, 134:1139-1144

29. Zeisel SH: Antioxidants suppress apoptosis. J Nutr 2004, 134:31793180

30. Janicke RU, Sohn D, Schulze-Osthoff K: The dark side of a tumor suppressor: anti-apoptotic p53. Cell Death Differ 2008, 15:959-976

31. Cheng WH, Zheng X, Quimby FR, Roneker CA, Lei XG: Low levels of glutathione peroxidase 1 activity in selenium-deficient mouse liver affect c-Jun N-terminal kinase activation and p53 phosphorylation on Ser-15 in pro-oxidant-induced aponecrosis. Biochem J 2003, 370: 927-934

32. Laine A, Topisirovic I, Zhai D, Reed JC, Borden KLB, Ronai Z Regulation of p53 localization and activity by Ubc13. Mol Cell Bio 2006, 26:8901-8913

33. Deininger MH, Meyermann R, Schluesener HJ: The allograft inflammatory factor-1 family proteins. FEBS Lett 2002, 514:115-121

34. Jia J, Bai Y, Fu K, Sun Z-J Chen X-M, Zhao Y-F: Expression of allograft inflammatory factor-1 and CD68 in haemangioma: implication in the progression of haemangioma. Clin Lab Invest 2008, 159:811-819

35. Autieri MV, Carbone CM: Overexpression of allograft inflammatory factor-1 promotes proliferation of vascular smooth muscle cells by cell cycle deregulation. Arterioscler Thromb Vasc Biol 2001, 21:14211426

36. Liu S, Tan WY, Chen QR, Chen XP, Fu K, Zhao YY, Chen ZW: Daintain AlF-1 promotes breast cancer proliferation via activation of the NFkB/cyclin D1 pathway and facilitates tumor growth. Cancer Sci 2008 , 99:952-957

37. Hunter T, Pines J: Cyclins and cancer II: cyclin D and CDK inhibitors come of age. Cell 1994, 79:573-582

38. Fu MF, Wang CG, Li ZP, Sakamaki T, Pestell RG: Cyclin D1: normal and abnormal functions. Endocrinology 2004, 145:5439-5447

39. Lin EY, NguYen AV, Russel RG, Pollard JW: Colony-stimulating factor 1 promotes progression of mammary tumors to malignancy. J Exp Med 2001, 193:727-739

40. Tricot G: New insights into role of microenvironment in multiple myeloma. Lancet 2000, 355:248-250

41. Gamberi G, Serra M, Ragazzini P, Magagnoli G: Identification of markers of possible prognostic value in 57 giant cell tumors of bone. Oncol Rep 2003, 10:351-356

42. Yang ZF, Ho DW, Lau CK, Lam CT, Lum CT, Poon RT, Fan ST: Allograft inflammatory factor-1 (AIF1) is crucial for the survival and pro-inflammatory activity of macrophages. Int Immunol 2005, 17 1391-1397

43. Kamada M, So A, Muramaki M, Rocchi P, Beraldi E, Gleave M: HSP27 knockdown using nucleotide based therapies inhibit tumor growth and enhance chemotherapy in human bladder cancer cells. Mol Cancer Ther 2007, 6:299-308

44. Romani AA, Crafa P, Desenzani S, Graiani G, Lagrasta C, Siamesi M, Soliani P, Borghetti AF: The expression of HSP27 is associated with poor clinical outcome in intrahepatic cholangiocarcinoma. BMC Cancer 2007, 7:232-241

45. Takayama S, Reed JC, Homma S: Heat shock proteins as regulators of apoptosis. Oncogene 2003, 22:9041-9047

46. Mosser DD, Morimoto RI: Molecular chaperones and the stress of oncogenesis. Oncogene 2004, 23:2907-2918

47. Garrido C, Brunet M, Didelot C, Zermati Y, Schmitt E, Kroemer G Heat shock proteins 27 and 70: antiapoptotic proteins with tumorigenic properties. Cell Cycle 2006, 5:2592-2601

48. Wang W, Eddy R, Condeelis J: The cofilin pathway in breast cancer invasion and metastasis. Nat Rev Cancer 2007, 7:429-440

49. Vydra J, Selicharová I, Smutná K, Šanda M, Matoušková E, Buršíková E, Prchalová M, Velenská Z, David Coufal D, Jiráček J: Two-dimensional electrophoretic comparison of metastatic and non-metastatic human breast tumors using in vitro cultured epithelial cells derived from the cancer tissues. BMC Cancer 2008, 8:107-119

50. Raftopoulou M, Hall A: Cell migration: rho GTPases lead the way. Dev Biol 2004, 265:23-32 